\title{
Parte II. Variables del ambiente hospitalario que inciden en el riesgo de infecciones de pacientes con cáncer y receptores de trasplante de precursores hematopoyéticos: Diseño, procesos asistenciales, calidad microbiológica del aire y agua
}

\author{
Ricardo Rabagliati ${ }^{1}$, Paula Catalán ${ }^{2}$, Marcela Rabello ${ }^{3}$, María Cristina Ajenjo y Marcela Zubieta ${ }^{4}$
}

'Departamento de Enfermedades Infecciosas del Adulto. Escuela de Medicina. Pontifica Universidad Católica de Chile. Santiago, Chile. ¿Unidad de Trasplante de Médula Ósea. Hospital Luis Calvo Mackenna. Santiago, Chile. 3Unidad de Infectología. Hospital Dr. Luis Calvo Mackenna. Santiago, Chile.

${ }^{4}$ Unidad de Oncología. Hospital Exequiel González Cortés. Fundación Nuestros Hijos. Santiago, Chile.

Los autores declaran ausencia de conflicto de interés.

El presente trabajo no recibió financiamiento alguno. Correspondencia a: Ricardo Rabagliati Borie rabagli@med.puc.c

\begin{tabular}{ll}
\hline Abreviaturas \\
AH & : ambiente hospitalario \\
$\mathrm{Al}$ & $:$ aspergilosis invasora \\
$\mathrm{AP}$ & $:$ ambiente protegido \\
$\mathrm{CA}$ & $:$ casa de acogida \\
$\mathrm{CPC}-\mathrm{IAAS}$ & $:$ Comité de Prevención y Control de Infecciones \\
& Asociadas a la Atención de Salud \\
$\mathrm{EFI}$ & $:$ enfermedad fúngica invasora \\
$\mathrm{EICH}$ & $:$ enfermedad de injerto contra hospedero \\
$\mathrm{HEPA}$ & $:$ High efficiency particulate air \\
IBI & $:$ infección bacteriana invasora \\
$\mathrm{NF}$ & $:$ neutropenia febril \\
$\mathrm{PHO}$ & $:$ pacientes hemato-oncológicos \\
$\mathrm{SARM}$ & $:$ Staphylococcus aureus resistente a meticilina \\
TPH: & $:$ trasplante de precursores hematopoyéticos \\
\end{tabular}

\section{Introducción}

L os pacientes con cáncer y receptores de TPH, que referiremos como pacientes hemato-oncológicos (PHO), transcurren largos y reiterados períodos de tiempo en el ambiente hospitalario (AH). Se entiende por $\mathrm{AH}$, todo lo que rodea al paciente: superficies (incluye la infraestructura y el mobiliario), aire, agua, alimentos, soluciones intravenosas (medicamentos, soluciones hidroelectrolíticas), personal y equipamiento clínico ${ }^{1}$.

El perfil de infecciones en $\mathrm{PHO}$ incluye bacterias como cocáceas grampositivas, bacilos gramnegativos, hongos como Candida spp, Aspergillus spp y otros hongos filamentosos; virus, parásitos, micobacterias tuberculosa y no-tuberculosas ${ }^{2-5}$. $Y$ tal como ocurre con otros pacientes, la presión selectiva ejercida por el uso indiscriminado de antimicrobianos, sumado a una baja adherencia a las prácticas de prevención de transmisión de infecciones, conlleva al incremento de agentes resistentes a diversos antimicrobianos, tales como Staphylococcus aureus resistente a meticilina (cloxacilina)-SARM ${ }^{6}$, Enterococcus faecium resistente a vancomicina ${ }^{7}$, bacilos gramnegativos multi-resistentes ${ }^{8-10}$ y Candida spp resistentes a azoles ${ }^{11}$.

La relación de causalidad entre patógenos identificado en el $\mathrm{AH}$ y enfermedad en el paciente no es fácil de evidenciar, requiriéndose que se cumplan varios criterios como: capacidad del microrganismo de sobrevivir y proliferar en el ambiente, demostración de la asociación de exposición y enfermedad en estudios retrospectivos, fuerza de asociación en estudios prospectivos, evidencia que la intervención en el ambiente logra erradicar el patógeno y la presentación de nuevos casos ${ }^{12}$. A pesar de estas dificultades de establecer causalidad en los casos puntuales, la evidencia acumulada permite afirmar que el 
AH tiene un rol como fuente de exposición a patógenos en la cadena necesaria para el desarrollo de infecciones en pacientes con alta susceptibilidad explicada por su profunda inmunosupresión por neutropenia y/o déficit en su inmunidad celular ${ }^{12}$.

Un riesgo ambiental muy conocido es la potencial exposición a Aspergillus spp., hongo filamentoso vehiculizado a través del aire, que da origen a aspergilosis invasora (AI), la causa más frecuente de enfermedad fúngica invasora $(\mathrm{EFI})$ en pacientes neutropénicos ${ }^{13}$. En nuestro país la frecuencia de EFI en niños que cursan neutropenia febril (NF) es de $6 \%$ de los episodios ${ }^{14}$ y en adultos está reportado hasta $20 \%$ de EFI en NF de pacientes hematológicos, de las cuales $80 \%$ corresponden a $\mathrm{AI}^{15}$.

Se debe precisar que la exposición a Aspergillus spp. no es únicamente en el $\mathrm{AH}$, sus conidias y las de otras especies de hongos filamentosos se encuentran ampliamente distribuidas en la naturaleza, asociados a zonas húmedas o con materia orgánica en descomposición. Su presencia en todo tipo de ambiente y su pequeño tamaño hace posible su transmisión por vía aérea, que no reviste importancia para hospederos inmunocompetentes, pero es relevante para toda aquella población de inmunocomprometidos, especialmente aquellos con neutropenia profunda y prolongada ${ }^{13}$. La inhalación de conidias de Aspergillus spp es particularmente preocupante en el AH donde los pacientes transcurren períodos de alta vulnerabilidad. El análisis del AH pone de manifiesto que la presencia de Aspergillus es extremadamente variable ${ }^{16,17}$, siendo reconocido que las actividades de remodelación o construcción incrementan su presencia ya que durante las obras se ponen al descubierto reservorios del hongo ${ }^{18}$. Otras fuentes de procedencia son los reservorios en sistemas de ventilación contaminados por polvo, el agua, la humedad en paredes, maderas, plantas o flores ${ }^{19}$. En los últimos años se han realizado estudios para cuantificar Aspergillus spp. en el AH; sin embargo, se debe señalar que no existe una clara definición que determine el punto de corte que signifique mayor riesgo de infección, aunque sí se ha logrado correlacionar que a mayor cuantía de Aspergillus spp. en el ambiente, medido a través de cultivos de aire ambiental, mayor es la incidencia de $\mathrm{AI}^{20-22}$.

Tal como se señalaba previamente, el agua, otro de los componentes relevantes del $\mathrm{AH}$, es otra fuente de infecciones nosocomiales ${ }^{23}$. El agua de beber puede contener microorganismos que representan poco riesgo para la mayoría de los pacientes; no obstante, en pacientes neutropénicos o receptores de TPH pueden causar infecciones graves. La contaminación de fuentes de agua hospitalarias por patógenos potenciales es relativamente común, si bien se trata de un problema conocido, no siempre es cuantificado por ausencia de protocolos de cultivo rutinarios que evidencien la situación. Las fuentes de agua, pueden llegar a ser reservorio de microorganismos patógenos, entre los que se encuentran bacterias (Pseudomonas aeruginosa, Legionella pneumophila, Acinetobacter spp, Klebsiella spp., Nocardia spp., Mycobacterium avium complex, etc.), parásitos (Cryptosporidium parvum, Giardia lamblia, Acanthamoeba spp., etc.) y hongos (Aspergillus spp., Fusarium spp. $)^{23-26}$, que bajo condiciones favorables de temperatura y nutrientes, pueden proliferar y alcanzar un hospedero susceptible, ya sea por ingestión de agua de consumo, contacto durante la higiene personal, al lavar o irrigar heridas o quemaduras, lavar equipos médicos como endoscopios, equipos dentales y catéteres o inhalación a través de nebulizadores o humidificadores ${ }^{27-31}$. Algunas características que favorecen la presencia de microorganismos en los sistemas de agua han sido estudiadas de manera especial en relación a Legionella spp. tales como presencia y crecimiento en estanques y biopelículas de cañerías, favorecido por temperatura no suficientemente elevada del agua caliente ${ }^{32}$. En la literatura médica internacional se han reportado múltiples brotes de infecciones nosocomiales en que se ha establecido una relación con las fuentes de aguas intrahospitalarias contaminadas con patógenos ${ }^{23-27}$.

El desafío para los hospitales es cómo ofrecer ambientes con el mínimo o nulo riesgo de adquirir infecciones, que a diferencia de la condición del paciente, teóricamente sí es posible modificar. Esto se traduce en la necesidad de identificar las mejores prácticas para que el $\mathrm{AH}$ sea seguro y no constituya un factor que aumente el riesgo de infecciones de pacientes profundamente inmunocomprometidos.

En este artículo se revisan aspectos del diseño e infraestructura, procesos asistenciales críticos, evaluación y recomendaciones para la calidad microbiológica del aire y agua de hospitales que atienden $\mathrm{PHO}$, orientados a disminuir la exposición y prevenir las infecciones en pacientes susceptibles.

\section{Infraestructura y diseño de las áreas de hospitalización}

Al revisar la literatura internacional publicada de estudios con investigación original que sustente las recomendaciones en el diseño de los servicios clínicos para la atención de $\mathrm{PHO}$, se reconoce que son escasos y existen pocas guías que intenten sistematizar los datos publicados $^{33-36}$.

Para dar respuesta al objetivo planteado se hace necesario abordar inicialmente los aspectos más generales del diseño, construcción o renovación de un centro hospitalario para la atención de PHO.

Para cualquier intervención mayor de un área hospitalaria se debe tener en cuenta las etapas que se requieren cumplir: planificación, diseño, proyectos de especialidades, construcción y, finalmente, certificación 
de la obra concluida ${ }^{37}$. Para proceder, resulta altamente recomendable conformar un grupo de trabajo multidisciplinario que incluya equipos clínicos expertos en el área oncológica e infecciones junto a ingenieros y arquitectos con experiencia en diseño y construcción de hospitales para que cada uno aporte con su visión, sin dejar de incluir desde un comienzo al respectivo Comité de Prevención y Control de Infecciones Asociadas a la Atención de Salud (CPC-IAAS) del hospital.

\section{Planificación}

Debe responder a los objetivos planteados para el espacio físico a construir o renovar, especificando si será para la atención de paciente con cánceres de órganos sólidos y/o hematológicos y/o TPH. En esta etapa resulta indispensable el dimensionamiento que tendrá el recinto para lo que se deben considerar datos demográficos y epidemiológicos de la población que será usuaria de este recinto, la frecuencia esperada de hospitalizaciones por requerimiento de quimioterapia o sus complicaciones, procedimientos diagnósticos o terapéuticos, estadías promedio según tipo de patología, entre otros factores. En caso de tratarse de un área dentro de un hospital general, debe precisarse el emplazamiento en su interior y su relación con otros servicios clínicos y con las unidades de apoyo que interactúan con mayor frecuencia con el área oncológica, debido al impacto que tendrá en los traslados de los pacientes, concurrencia de tratantes, interconsultores y profesionales de apoyo, así como en los flujos de muestras, fármacos, hemocomponentes y apoyo nutricional, entre otros.

\section{Diseño}

En esta etapa, se lleva a planimetría lo discutido en la etapa previa. En el diseño general, debe velarse por el cumplimiento de las regulaciones sanitarias vigentes. Se debe definir tipo y características de las habitaciones y baños, número de habitaciones con ambiente protegido (AP), salas de procedimientos, espacios comunes y áreas de apoyo, entre otras. Se debe incluir detalles de diseño, mobiliario, aspectos funcionales, circuitos para los pacientes, flujos del personal clínico y recorridos de las visitas.

\section{Proyectos de las diferentes especialidades}

Contempla el diseño por expertos de las instalaciones sanitarias y eléctricas, gases clínicos, climatización y puntos de conexión informática.

\section{Construcción}

Durante esta fase, es muy importante la verificación por parte de inspectores técnicos que las obras se desarrollan según el diseño planificado y avanza según los plazos establecidos. Del punto de vista de la prevención de infecciones, se debe prestar particular atención a aquellos casos que corresponden a renovaciones de espacios físicos de áreas oncológicas de hospitales que se encuentran en funcionamiento, asegurando la implementación de medidas de mitigación de la contaminación por polvo del AH durante el desarrollo de la obra, aspecto que se comenta más adelante en la sección de procesos críticos.

\section{Certificación de la obra concluida}

En esta etapa se requiere confirmar que la construcción cuenta con las especificaciones planificadas y se encuentra en condiciones de entrar en operación. El CPC-IAAS local debe certificar de su punto de vista las condiciones seguras de las instalaciones y cumplir los aspectos regulatorios correspondientes antes de la entrada en funcionamiento.

\section{Definición de área oncológica}

Para la atención de los PHO en sus cuidados básicos, es altamente recomendable diseñar áreas diferenciadas de otras áreas de atención de pacientes con patologías médicas o quirúrgicas generales. Su ubicación dentro del hospital debe considerar desplazamientos relativamente simples y breves a las áreas de estudios de imágenes, salas de procedimientos y áreas de cuidados críticos. A su vez, las unidades de apoyo de farmacia, laboratorio y banco de sangre deben estar a una distancia relativamente cercana o disponer de mecanismos que aseguren respuesta rápida de despacho o traslado de requerimientos.

La definición del área oncológica como un área exclusiva, se basa en la facilitación de la estandarización de los cuidados. En primer lugar, se debe tener en cuenta que de esta manera se concentra la atención de pacientes inmunocomprometidos por su enfermedad y su tratamiento. En segundo lugar, otro beneficio radica en que a esta área confluyen varias especialidades médicas, que deben interactuar entre sí, para la apropiada atención de los pacientes desde el diagnóstico inicial, tratamiento, manejo de complicaciones y cuidados paliativos ${ }^{38-40}$. Otra razón que justifica el área oncológica, es el innegable beneficio de la concentración de atención de enfermería especializada que garantiza la experiencia en la administración correcta de quimioterapia, preparación frente a las complicaciones más frecuentes, incluyendo las infecciones y su tratamiento, junto a una estricta adherencia a las normas de prevención y control de infecciones. Finalmente, se reúne en un área grupos de PHO y familias en condiciones de alto estrés psicológico por el diagnóstico oncológico lo que requiere velar con especial cuidado por las condiciones de un ambiente terapéutico de tranquilidad.

Para el adecuado funcionamiento de esta área, resulta de utilidad generar criterios de ingreso y las excepciones para permitir el ingreso de otros pacientes, lo que facilita las decisiones cuando la demanda por camas de hospitalización es elevada. 
En relación a la necesidad de los PHO de cuidados de mayor complejidad como intensivos e intermedios, si bien puede tener algunos beneficios incluirlas como una sección dentro de las áreas oncológicas generales, resulta más costo efectivo considerarlas dentro de las unidades de paciente crítico en funcionamiento del hospital, para lo cual se hace necesario que cumplan los mismos requisitos básicos de unidades para pacientes oncológicos, especialmente contar con unidades de AP para los $\mathrm{PHO}$ que lo requieran cuando se trasladen a este tipo de unidades.

\section{Tipo de habitaciones}

En relación a la necesidad de requerir habitaciones compartidas o individuales, la literatura científica no señala evidencia clara de una sobre otra. Sí existe la recomendación que, para cualquier hospital, el diseño de habitaciones individuales favorece la flexibilidad, podría tener menores costos de operación y dan más comodidad a pacientes, su familia y al personal ${ }^{39,40}$.

Van de Glind y cols $^{41}$, revisaron los atributos de la habitación individual como dignidad y privacidad, satisfacción, ruido y calidad del sueño, tasa de infecciones y aspectos de seguridad de pacientes, sin lograr identificar de manera consistente la menor frecuencia de infecciones con el uso de habitaciones individuales; sin embargo, esta revisión presenta limitaciones ya que no se incluyen estudios caso control, centros con endemia de bacterias resistentes a antimicrobianos ni estudios en hospitales de pacientes oncológicos, sino que hospitales generales. En otro estudio, de tipo cualitativo, sobre la percepción de los pacientes en relación a habitación individual o compartida, ellos destacan los beneficios de la habitación compartida cuando se encuentran en mejores condiciones y habitaciones individuales cuando están más enfermos ${ }^{42}$. Teóricamente se puede plantear que en la medida que exista un menor número de pacientes por habitación, existe una menor exposición a otros pacientes, personal y visitas de manera simultánea, disminuyendo el riesgo de adquirir eventuales enfermedades transmisibles. La elección de diseños de infraestructura de pieza de uso individual debe incluir baño para uso exclusivo del paciente por cada habitación. En la medida que el número de personas por habitación y por baño sea menor, disminuye el riesgo de transmisión de infecciones por compartir espacios comunes y artefactos sanitarios; sin embargo, la evidencia no es clara en demostrar que la habitación de uso individual necesariamente disminuya el riesgo de infecciones asociadas a la atención de salud ${ }^{43-46}$. Se deben considerar las condiciones de los pacientes y la epidemiología del centro $^{43}$. Bracco y cols., demostraron que en un centro sin hiperendemia de SAMR, las habitaciones individuales contribuyen a disminuir la adquisición de infecciones por este agente $^{47}$, en la misma línea se ha demostrado que el uso de habitaciones individuales en unidades de cuidados intensivos disminuye la adquisición de infecciones por SARM y Costridioides difficile ${ }^{48}$.

Por lo tanto, con los datos presentados, nos parece adecuado recomendar de preferencia habitación individual para la hospitalización de pacientes en el área de PHO. En caso de existir restricciones, que no permiten considerar habitaciones individuales para todos los pacientes, nuestra recomendación es que al menos deben considerarse habitaciones individuales para pacientes con mayor riesgo de infecciones tales como aquellos con leucemia aguda u otros cánceres con esquemas de quimioterapia de alta intensidad, receptores de TPH, ya sea neutropénicos previo a la implantación, o en los seis meses siguientes o aquellos con enfermedad injerto contra hospedero (EICH) con uso de fármacos inmunosupresores.

\section{Ambiente protegido}

Este tipo de habitaciones busca garantizar aire libre de patógenos, especialmente conidias de hongos filamentosos. Se entiende como AP a habitaciones individuales, con presión positiva al interior de la unidad respecto al exterior y al pasillo $\geq 2,5$ Pascales (es decir $\geq 0,002 \mathrm{~cm}$ de mercurio $\mathrm{o} \geq 0,025 \mathrm{~cm}$ de agua), más de 12 recambios de aire por hora que ingresa a través de filtros de aire de alta eficiencia, HEPA (High efficiency particulate air, por su sigla en inglés) que logran evitar el paso de 99,97\% de las partículas sobre 0,3 micrones que garantiza óptima calidad de aire (Figura 1). Los materiales de construcción

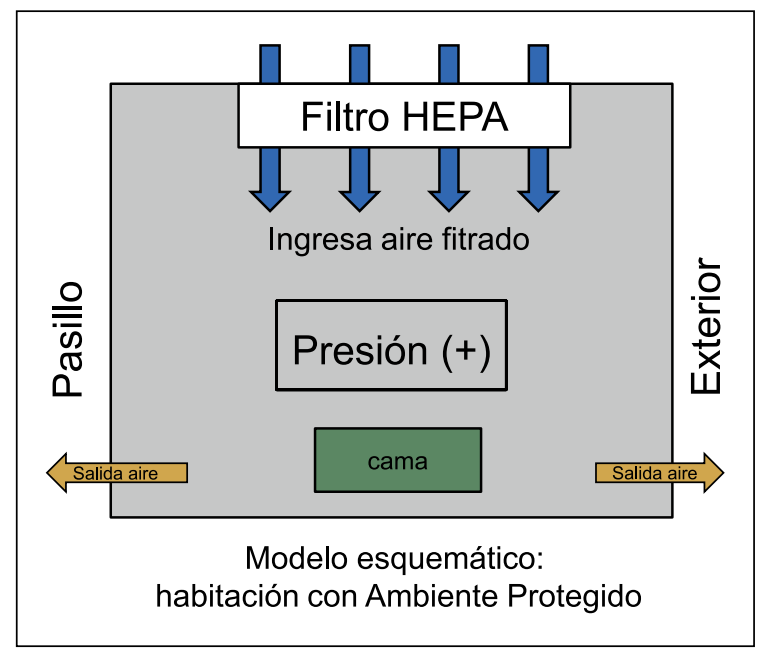

Figura 1. Esquema de "Ambiente protegido": Aire ingresa a la habitación filtrado a través de filtro HEPA, al interior se recambian los volúmenes de aire al menos 12 veces por hora y se mantiene presión positiva $\geq 2,5$ Pascales respecto a pasillo y exterior. (reproducido con autorización de autor desde: Rabagliati R. Actualización en el diagnóstico y manejo de aspergilosis invasora en pacientes adultos. Rev Chilena Infectol 2018; 35: 531-544) 
deben asegurar que no deben expeler polvo, asegurar superficies lisas evitando la acumulación de partículas, ser fácilmente lavables y resistentes al uso de antisépticos y desinfectantes para garantizar la máxima higiene de la habitación ${ }^{12,33,49}$. Los filtros deben ser cambiados periódicamente según la recomendación del manufacturador. La exclusa no es indispensable como parte del diseño del $\mathrm{AP}^{33}$.

Tal como se comentaba previamente, el AP debe considerarse no sólo en las áreas de oncología de menor complejidad, sino que también debe implementarse al interior de los sectores de cuidados intensivos e intermedios, a fin de garantizar que los pacientes que así lo requieren mantengan satisfecho el requerimiento de aire seguro durante todas las fases de atención intrahospitalaria. Incluso no se debe descuidar el traslado de los pacientes al salir de una habitación con AP, oportunidad en que deben portar mascarilla de alta eficiencia N95 ${ }^{12}$

En caso de no disponer suficientes habitaciones de AP, se debe priorizar aquellos de mayor riesgo (p. ej.: neutropenia más profunda y prolongada) a estas habitaciones y aquellos pacientes con relativo menor riesgo disponer de medidas alternativas como el uso de filtros portátiles que logran disminuir la posibilidad de exposición a conidias de hongos, aunque no se ha demostrado que sean equivalentes a las habitaciones de $\mathrm{AP}^{33}$.

En general, no debería apagarse el sistema de filtración de aire de las habitaciones con AP, ya que el reinicio podría significar un incremento transitorio de las conidias del ambiente expelidas por los sistemas de ventilación.

No se recomienda en caso alguno revertir las presiones para convertir una habitación con AP a una con precaución respiratoria; si se llegara a dar el caso que un paciente presente ambos requerimientos, es preferible que se hospitalice en una habitación individual, con presión neutra, en que se utilice un filtro HEPA portátil. Si esta fuera una condición frecuente, se puede incorporar una habitación con diseño especial, que dé respuesta simultánea a ambos requerimientos, que incluye necesariamente una exclusa, ya sea con presión positiva respecto habitación y pasillo o al contrario la exclusa con presión negativa respecto a habitación y pasillo. La ventaja del primer diseño es que el equipo tratante puede utilizar la exclusa para la postura de los elementos de protección personal; de lo contrario, debería ingresar a la exclusa con mascarilla ${ }^{50}$.

\section{Unidades de apoyo}

El diseño y planificación de hospitales para atención de $\mathrm{PHO}$ debe considerar unidades de apoyo críticas para el manejo de estos pacientes, garantizando entrega de respuesta inmediata para requerimientos críticos y en plazos definidos para los restantes. Entre ellas, las más relevantes para el diagnóstico y manejo de los problemas infecciosos de los PHO, se encuentran:

- Anatomía Patológica, incluyendo especialista y técnicas diagnósticas de enfermedades infecciosas.

- Banco de Sangre, adicional a todo el soporte transfusional e infusión de precursores; debe poder dar respuesta al ocasional requerimiento de transfusión de granulocitos.

- Farmacia, con disponibilidad de antimicrobianos de uso más frecuentes y mecanismos de acceso a fármacos de menor frecuencia de uso o ausentes del arsenal local (se revisa en detalle en el Capítulo III).

- Imagenología, debe contar con todas las prestaciones de radiografía, ecografía, TAC, RM, medicina nuclear, PET, con oportunos reportes por radiólogos con experticia en PHO y con posibilidad de realizar procedimientos diagnósticos de radiología intervencional.

- Laboratorio, con desarrollo avanzado para diagnóstico etiológico (bacterias, micobacterias, hongos, parásitos y virus), evaluación de susceptibilidad in vitro según corresponda, serologías diagnósticas y determinación de concentraciones plasmáticas de antimicrobianos (se revisa en detalle en el Capítulo III).

\section{Disponibilidad de especialidades médicas}

Se debe asegurar acceso expedito a diferentes especialistas o sub-especialistas de adultos o niños, según corresponda, para evaluación de los pacientes en todo momento de su evolución (horarios hábiles y no hábiles). Idealmente se deben conformar equipos de trabajo de infectólogo y hematólogo/oncólogo vinculados por el interés y la experiencia en el diagnóstico y manejo de infecciones en pacientes inmunocomprometidos.

Sin duda, considerando la frecuencia de las complicaciones infecciosas, el especialista en enfermedades infecciosas debe ser parte del equipo de atención de los PHO; su rol es muy importante para definir estrategias de prevención, establecer y/o validar protocolos diagnósticos y terapéuticos para los problemas más frecuentes. Es recomendable que esté disponible para evaluar aquellas situaciones de pacientes individuales que en su complejidad se desvían de las complicaciones habituales o de los plazos habituales.

\section{Calidad asistencial: Definición de procesos seguros}

Los procesos asociados a la atención del $\mathrm{PHO}$, en el ámbito relacionado a la prevención, diagnóstico y terapia de las infecciones, deben ser conocidos en detalle y la aplicación de mejoras debe ser materia de permanente evaluación. 
Actualmente, en nuestro país se exige que los hospitales que atienden pacientes con cáncer cuenten con acreditación de calidad otorgada por la Superintendencia de Salud. En su Manual vigente exige el cumplimiento de los siguientes estándares relacionados al cuidado del paciente oncológico $^{51}$ : decisiones de tratamiento oncológico; condiciones de seguridad en la preparación, almacenamiento, distribución y desecho de medicamentos antineoplásicos; protocolos actualizados, sistemas de registros específicos y supervisión de las prácticas relacionadas con la quimioterapia; y vigilancia de las infecciones asociadas a catéteres vasculares centrales. Esto se entiende como el mínimo exigible; a nuestro juicio, deberían agregarse otros aspectos relevantes en el ámbito de la prevención y manejo de infecciones de los $\mathrm{PHO}$, incluyendo políticas y procedimientos definidos, indicadores de cumplimiento y seguimiento en los siguientes ámbitos:

\section{Prevención de infecciones}

- Capacitación mandatoria y actualización frecuente en IAAS, focalizada en los riesgos particulares de los PHO.

- Preparación anual para el período de elevación anual de infecciones por virus respiratorios a fin de aminorar su transmisión, incluyendo vacunación obligatoria de influenza del personal y mecanismos para la exclusión de personal enfermo por alguno de estos virus.

- Procedimiento de intervención inmediata en contactos de pacientes, visitas o personal con varicela, coqueluche, influenza, virus respiratorio sincicial, tuberculosis, entre otros.

- Procedimiento de segregación de PHO en servicio de urgencia respecto a otros enfermos potencialmente portadores de enfermedades infecto-contagiosas.

- Mitigación de riesgo de exposición a polvo ambiental en relación a construcciones, remodelaciones, mantenimiento.

- Programas de control microbiológico de aire y agua.

- Programas de mantención de habitaciones con AP.

- Plan de vacunación contra varicela del personal o documentación de inmunidad específica.

\section{Del punto de vista de la operación clínica}

- Criterios de ingreso a unidades de oncología y habitaciones con AP.

- Traslados internos seguros de PHO desde sus habitaciones con AP.

\section{Apoyo diagnóstico}

Protocolos de enfrentamiento diagnóstico en diferentes escenarios para demostrar etiología infecciosa con la mayor precisión posible, incluyendo laboratorio de microbiología, biología molecular, imagenología y anatomía patológica. La definición de la disponibilidad de pruebas diagnósticas en los hospitales que atienden PHO se desarrolla en el Capítulo II.

\section{En el ámbito terapéutico}

Guías de terapia para las infecciones más frecuentes, que incluya el arsenal mínimo requerido, los tiempos de administración desde el diagnóstico, períodos de tratamiento. Este aspecto se desarrolla en el Capítulo III.

Establecer definiciones claras de diagnóstico y terapia pueden lograr impacto significativo en los resultados del manejo de casos, como se destaca en la experiencia publicada de Hospital de Clínicas de Porto Alegre, Brasil, quienes luego de implementar una vía clínica estandarizada en NF lograron demostrar reducción de mortalidad $(24,4$ a $14,4 \% ; p=0,017)$ con una adherencia cercana a $70 \%$. Uno de los elementos que debe ser vigilado es el tiempo de administración de antimicrobianos desde el diagnóstico de NF, que no debería ser mayor a $2 \mathrm{~h}$; se ha demostrado que este factor juega un rol crítico, cada hora que se retrasa su administración, la estadía de los pacientes se prolonga en $8 \mathrm{~h}^{53}$.

\section{Calidad microbiológica del aire}

La implementación de AP en los hospitales de nuestro país que atienden $\mathrm{PHO}$ es heterogénea. Si bien existen algunos hospitales en que se encuentra implementado según su definición, existen varios otros en que se realiza alguna intervención parcial y otros con ninguna medida de prevención. En un estudio aún no publicado de Rabello y cols., se investigó la calidad microbiológica del aire en unidades oncológicas de los hospitales públicos que atienden niños con cáncer de la Región Metropolitana constatando un número elevado de hongos potencialmente patógenos en todas las áreas de los centros sin intervención de aire.

Existen diferentes recomendaciones en la literatura médica internacional ${ }^{12,33,49}$, sobre las intervenciones del aire ambiental, que dependen fundamentalmente del tipo de paciente y su riesgo de AI. A mayor riesgo de AI se debe exigir la máxima calidad de aire ambiental, es decir AP, según lo definido previamente, para pacientes con neutropenia $<100$ céls $/ \mathrm{mm}^{3}$ con duración esperada de más de siete días como suele ocurrir en pacientes que reciben quimioterapia para inducción o reinducción de leucemias agudas, TPH previo a la implantación u otros pacientes que reciben esquemas de alta intensidad.

Respecto al seguimiento y control de la calidad microbiológica del aire del AH, y los AP en particular, ésta se puede constatar mediante la determinación de la carga fúngica a través del cultivo de aire ambiental, con 
la ayuda de implementos específicos que concentran el aire del recinto sembrándolo directamente en placa. Sin embargo, se debe comentar que no ha sido establecido el real aporte de la vigilancia rutinaria de la contaminación ambiental por hongos con el objetivo de prevenir AI, debido a la ausencia de estandarización de su técnica (número de muestras, intervalos, sectores específicos a medir, volumen de aire a evaluar, entre otros), periodicidad de la medición, puntos de corte asociados a riesgo, dificultad en la interpretación de resultados (falsos negativos, baja sensibilidad, tiempos prolongados para obtener resultados $)^{12} \mathrm{y}$ además, porque la carga fúngica del aire ambiental es un fenómeno dinámico asociada a múltiples variables ${ }^{12,33,49}$.

Por lo tanto, no es posible realizar una recomendación sobre el monitoreo rutinario y sistemático de la calidad microbiológica del aire en beneficio de generar un $\mathrm{AH}$ que ofrezca la mayor seguridad posible ${ }^{54}$. A pesar de esta limitación, cada hospital puede desarrollar protocolos de control periódico de aire ambiental, a fin de generar su propia experiencia teniendo presente las dificultades señaladas; la evidencia disponible no nos permite entregar una recomendación específica sobre su ejecución, pero se podría considerar realizar control microbiológico del aire ambiental después de completar las mantenciones periódicas o posterior a los cambios de filtros HEPA o en caso de situación de brote. Sin embargo, lo que sí recomendamos es que cada centro debe minimizar los riesgos a través de adecuados procedimientos de mantención, con su respectiva supervisión, de los sistemas de ventilación, los recambios de aire, las presiones diferenciales y los filtros HEPA, tanto portátiles como de los AP.

Para aquellos centros que definen realizar vigilancia de la calidad microbiológica del aire ambiental, se debe asociar a un plan de manejo de acuerdo a los resultados. Respecto a la muestra de aire de AP, debe obtenerse altos volúmenes de muestras de aire de 1.000 litros $\left(1 \mathrm{~m}^{3}\right)^{55}$. Existe acuerdo que en áreas con AP, al cultivar el aire se espera ausencia de desarrollo de hongos, un resultado desfavorable corresponde a aquellos con carga fúngica de 1 o más ufc $/ \mathrm{m}^{3}$ de hongos patógenos ${ }^{13}$. En casos de resultado desfavorable se debe dejar de utilizar la unidad como AP hasta que no se verifique el adecuado funcionamiento de los sistemas de presión y recambio de aire, sellos, indemnidad del filtro HEPA, aseos y fuentes fúngicas no detectadas. Una vez resueltas estas situaciones, se debe tomar un nuevo cultivo de aire ambiental que demuestre la ausencia de hongos patógenos para poder reiniciar el uso de la habitación como AP.

\section{Recomendaciones}

En general, las medidas preventivas recomendadas para conseguir y mantener en los hospitales un adecuado nivel de bioseguridad del aire ambiental son las siguientes:
- Asegure el adecuado uso y mantenimiento de las habitaciones con AP:

- Mantenga la puerta cerrada.

- En caso de contar con exclusa evite abrir ambas puertas de manera simultánea.

- Chequeo de presión positiva y recambios de aire.

- Cambio periódico de filtros hepa.

- Criterios de uso de AP para pacientes de mayor riesgo de AI y medidas de continuidad de la protección de pacientes al trasladarse desde su habitación de AP hacia otras unidades o áreas de exámenes con uso de mascarillas N95 ${ }^{56}$.

- Asegure el adecuado mantenimiento de la infraestructura de las áreas con y sin AP: indemnidad de paredes, cielos, puertas y sellos.

- Vele por el adecuado cumplimiento de las normas de aseo de la habitación evitando levantar polvo ambiental. Es reconocido que si éste no es realizado de manera cuidadosa, evitando que se genere polvo ambiental, se incrementan los aerosoles conteniendo Aspergillus $\mathrm{spp}^{57}$.

- Prohibir flores y plantas dentro de la habitación ${ }^{49,58}$.

- Cumplimiento estricto de las políticas de renovación, remodelación de áreas clínicas, tomando todas las medidas para asegurar el aislamiento de las zonas sometidas a remodelaciones, con sellos que impidan el paso de polvo a las áreas de pacientes ${ }^{12,33,49,59-62}$.

- Definición local sobre el uso y periodicidad de vigilancia microbiológica de la contaminación fúngica del aire ambiental, teniendo presente las limitaciones de los métodos vigentes; la falta de evidencia para la interpretación de los resultados y el alto costo que conlleva su implementación.

- Vigilancia de casos de infecciones por filamentosos para detectar oportunamente situaciones de brote.

\section{Calidad microbiológica del agua}

Los sistemas de distribución de agua en hospitales son redes comúnmente complejas y extensas. Los microorganismos involucrados en su contaminación, dado su nicho ecológico, son de una gran ubicuidad, tal como se señalaba más arriba; entre ellos se encuentran bacterias, parásitos y hongos. Su crecimiento y mantención en el sistema se ve favorecido por los puntos muertos, la formación de biopelículas en las cañerías, los flujos bajos de agua, la temperatura insuficiente (bajo $55^{\circ} \mathrm{C}$ en las cañerías de agua caliente y sobre $20^{\circ} \mathrm{C}$ en cañerías de agua fría) y las salidas de agua de uso poco frecuente, factores que hacen casi imposible la erradicación completa de los microorganismos ${ }^{63-67}$.

Los mecanismos por los cuales los patógenos presentes en el agua pueden afectar a los PHO son: contacto directo, 
ingestión de agua, contacto indirecto a través de algún dispositivo, inhalación de aerosoles y aspiración de agua contaminada. Los tres primeros se asocian a bacilos gramnegativos y micobacterias no tuberculosas; en cambio, los aerosoles son la forma de ingreso de Legionella sp. al tracto respiratorio ${ }^{68}$.

La estrategia de control de patógenos transmitidos a través del agua debe apuntar a la reducción del número de agentes patógenos y la protección de los $\mathrm{PHO}$ de alto riesgo. Se han usado múltiples métodos para reducir la posibilidad de contaminación microbiológica de los sistemas de distribución de agua ${ }^{69-79}$, tales como evitar áreas de agua estancadas por bajo flujo o uso limitado, hacer correr regularmente el agua de las llaves y mantener el agua caliente y fría a la temperatura correcta (la caliente sobre $55^{\circ} \mathrm{C}$ y la fría a menos de $20^{\circ} \mathrm{C}$ ), incluso incrementando transitoriamente la temperatura del agua caliente a $66^{\circ} \mathrm{C}$ hasta el punto de salida, y aplicando cloración al agua para que se distribuya en la red ${ }^{12}$. Otras medidas que se han utilizado son el uso de filtros en los puntos de entrada del agua al edificio o los puntos finales de salida, calentamiento tipo pasteurización y flushing del sistema, desinfección mediante luz ultravioleta, uso de sistemas recubiertos con cobre y plata ionizada, uso de materiales que no sirvan de sustrato para la formación de biopelículas, uso de desinfectantes en tipo y concentración adecuados para evitar la corrosión de los materiales y vaciamiento de los sistemas de acopio e incorporación de filtros. A pesar de este importante número de medidas, no existen recomendaciones consensuadas universales ${ }^{79}$.

Respecto al control microbiológico del agua hospitalaria, tampoco existe consenso internacional; sin embargo, se debe destacar la guía técnica vigente en Francia desde 2005, que basada en criterios de seguridad, recomienda control a lo menos cuatro veces al año en los hospitales, que incluya monitoreo de la presencia de Legionella spp., Pseudomonas y coliformes. Para unidades que atienden pacientes con compromiso inmunológico profundo, receptores de trasplantes y usuarios de corticosteroides, recomienda procurar concentraciones no detectables para Legionella sp y < $1 \mathrm{ufc} / 100 \mathrm{ml}$ para coliformes totales y P. aeruginosa ${ }^{80}$. En nuestro país no existen normativas específicas sobre la concentración de microorganismos en las fuentes de aguas hospitalarias ni tampoco guías para prevenir las infecciones intrahospitalarias transmitidas por el agua; solamente se encuentra normado el requerimiento de ausencia de contaminación bacteriana del agua medida por presencia de coliformes (incluye E. coli, Klebsiella sp., Enterobacter sp. y Citrobacter sp, entre otros) ${ }^{81}$.

A nuestro conocimiento, la información nacional disponible del agua en hospitales de PHO lo entrega un reciente estudio efectuado por parte de nuestro grupo de trabajo de la calidad microbiológica y química del agua en cuatro Unidades de Oncología Pediátrica del Sistema Público de Salud de la Región Metropolitana, en el que se evaluó la presencia de diferentes agentes en las torres de agua y en la que llega a las habitaciones de los pacientes en llaves de lavamanos y duchas, demostrándose la presencia de microorganismos en $50,1 \%$ de las muestras, identificándose parásitos en $71 \%$ (Microsporidium), seguido por bacterias en $32 \%$ (Pseudomonas sp, Enterobacter sp., Alcaligenes sp., Legionella sp., entre otros) y hongos en $17 \%$ de las muestras (Fusarium spp, Acremonium spp., Rhizopus spp. y Rhodotorula spp. $)^{82}$. Estos resultados enfatizan la necesidad de generar más información local y establecer estrictas medidas para limitar la exposición de pacientes de alto riesgo a agentes potencialmente patógenos transmisibles a través del agua.

\section{Recomendaciones}

- Asegure un plan para mantener un adecuado funcionamiento de los sistemas de acopio (estanques), distribución y temperatura del agua caliente (sobre $55^{\circ} \mathrm{C}$ ) y fría (bajo $20^{\circ} \mathrm{C}$ ).

- En situación de brote de infección bacteriana, fúngica o parasitaria, incluya el agua como fuente a investigar, tanto agua de los estanques de acumulación, como del agua de la llave de lavamanos y ducha, si bien se debe ser muy cauto en la interpretación de resultados.

- Por el momento, no recomendamos incorporar de manera rutinaria el estudio del agua para la búsqueda de Legionella sp., Pseudomonas sp., Mycobacterium sp., hongos y parásitos. Se requiere contar con mayor evidencia sobre la interpretación de los resultados y sus reales beneficios. Existen una serie de puntos no bien definidos en el estudio microbiológico del agua: no es claro cuáles son las muestras más adecuadas, los métodos de obtención, su periodicidad, técnicas de estudio microbiológico a incluir, ni los puntos de corte que determinen el mayor riesgo y las medidas efectivas de intervención, por lo que parece más adecuado contar con más información antes de generar recomendaciones que pueden generar alto costo con poca posibilidad de intervención.

- Cuando las llaves de agua dejan de utilzarse por varios días o semanas, deje correr el agua de la llave de manera periódica, o al menos previo al ingreso de pacientes a la habitación.

- Use agua estéril para limpieza de insumos o equipos que toman contacto con vías aéreas o sistema digestivo de los pacientes

- En los pacientes con neutropenia, emplee agua envasada para beber, higiene bucal y lavado de dientes. Respecto a la higiene corporal: Considere baño de esponja con agua estéril o incorporar filtros en los grifos de salida del agua de la ducha. 


\section{Continuidad del cuidado al alta: Casas de acogida}

Las casas de acogida (CA) nacen en respuesta a la necesidad de recibir al enfermo que no puede regresar a su hogar, por distintas razones, luego del alta hospitalaria, a fin de permanecer a corta distancia de los hospitales referentes para asegurar sus controles frecuentes y/o continuidad de tratamiento.

La información respecto a control de infecciones ha sido desarrollada desde sus orígenes en el ambiente hospitalario, pero no en el cuidado ambulatorio, o de transición, donde se ha planteado la necesidad de estudiarla en profundidad debido a importantes carencias de información ${ }^{83}$. Si bien desde hace varios años se ha identificado que la problemática existe y con potencialidad de incrementarse ${ }^{84}$, los esfuerzos inciales en estandarizar la vigilancia y prevención no ha abordado todas las áreas de cuidado ambulatorio ${ }^{85-87}$.

En la literatura médica internacional se pueden encontrar publicaciones relacionadas a regulaciones o recomendaciones respecto a la organización, control de infecciones y acreditaciones de las instituciones de cuidados de largo término, hospitalización domiciliaria o cuidados en casa ${ }^{88-92}$, pero no abordan específicamente estructuras de transición del cuidado como las CA para enfermos de cáncer.

A pesar de que no existen recomendaciones específicas que se hagan cargo de estos puntos, nos parece relevante dar directrices generales para dar continuidad a la seguridad exigida a los centros hospitalarios en la prevención de exposición a patógenos durante la estadía en estas unidades. En consideración que las CA pueden hospedar a varios pacientes simultáneos, con diferente historial infectológico, a nuestro juicio deben ser consideradas como una extensión del hospital en término de las estrategias generales para evitar la adquisición de infecciones cruzadas entre sus huéspedes. Algunos aspectos particulares de las CA que requieren ser normados a fin de disminuir el riesgo de exposición y colonización de agentes asociados a la atención de salud son los siguientes:

- Tipo de habitaciones: Considerando que el historial de infecciones y colonizaciones suele ser diferente para cada paciente que ingresa en la CA, se debe disponer de habitaciones para uso exclusivo del binomio paciente y acompañante, con su baño de uso exclusivo. La materialidad y mobiliario de la habitación debe facilitar la limpieza de las instalaciones, asegurando procedimientos de aseo al momento de la salida de paciente y llegada de un nuevo paciente.

- Espacios comunes: También se debe tener en cuenta los aspectos de materialidad y mobiliario para facilitar procesos de aseo. Tener procedimientos definidos para evitar el uso simultáneo de estas áreas por pacientes portadores de agentes multi-resistentes.

- Manejo de pacientes con portación de agentes infecciosos multi-resistentes. Precauciones adicionales a precaución estándar: Todo paciente con confirmación de portación de agentes infecciosos que requiriese algún tipo de precaución (respiratorio, de contacto o gotitas) al momento del alta hospitalaria, debe mantenerse al ingreso a la CA. Se debe tener presente que la portación de SARM, Enterococcus resistente a vancomicina (ERV), bacilos gramnegativos resistentes y $C$. difficile puede mantenerse por períodos variables y alcanzar hasta varios meses por lo que resulta indispensable su identificación para la implementación de las precauciones correspondientes. Por lo tanto, se requiere establecer un procedimiento de solicitud de información actualizada de los microorganismos identificados durante su hospitalización.

- Educación en higiene de manos y supervisión de su cumplimiento.

- Procedimiento frente a demolición, construcción y mantención: Se debe crear un protocolo de acciones frente a demoliciones, construcciones y mantenciones que afecten, tanto a la casa de acogida como sus alrededores. El objetivo es evitar las infecciones asociadas a la exposición a polvo en el aire ambiental. Como base, se puede utilizar recomendaciones vigentes en construcciones en hospitales ${ }^{61,62}$.

- Regulación de visitas: Se debe contar con protocolos que regulen su número, frecuencia y horarios, evitando la sobrepoblación con el consecuente riesgo de infecciones. Se debe fomentar la importancia de la higiene de manos, facilitando dispositivos de alcohol gel y exigiendo que personas con síntomas respiratorios, digestivos, erupciones cutáneas o fiebre no deben ingresar a estas dependencias.

- Capacitación del personal: El número y rol de quienes se desempeñan en la CA dependen del tamaño de la unidad, pero habitualmente debe incluir enfermera y técnico de nivel superior en salud-TENS, personal administrativo y de apoyo. Deben definirse algunos requerimientos mínimos, como capacitación en prevención de infecciones, vacunación anual contra influenza y asegurar la inmunidad contra varicela y hepatitis B.

\section{Conclusiones}

En resumen, el $\mathrm{AH}$ es una fuente común de riesgo para PHO de adquirir infecciones en su etapa de mayor vulnerabilidad. Por lo tanto, se deben tomar las precauciones necesarias desde el adecuado diseño de las unidades de 
oncología, incluyendo habitaciones con AP para los $\mathrm{PHO}$ con mayor riesgo de AI, asegurar un oportuno acceso a especialistas, a unidades de cuidado crítico y áreas de apoyo clínico. Asegurar la definición de procesos asistenciales con vigilancia de su cumplimiento, en particular medidas de prevención de exposición a patógenos en relación a aire ambiental o el agua para consumo o baño. Y en ausencia de consenso, tener presente la opción de evaluar la carga microbiológica del aire y agua como una forma de determinar el riesgo local y la vigilancia del funcionamiento de las medidas preventivas. Finalmente, comprender las CA como una extensión del $\mathrm{AH}$, por lo que se deben regular los aspectos generales de infraestructura y operación, a fin de seguir garantizando un espacio seguro para $\mathrm{PHO}$ en la perspectiva de evitar la exposición a patógenos.

\section{Resumen}

El ambiente hospitalario es una fuente potencial de exposición a patógenos como bacterias, hongos y parásitos, que pueden provocar infecciones en pacientes con cáncer incluyendo receptores de trasplante de precursores hematopoyéticos. Para aminorar este riesgo, se deben tener en cuenta los elementos de diseño, construcción y emplazamiento del área de atención de pacientes. Se entregan recomendaciones para proveer ambientes seguros, incluyendo características y uso de ambiente protegido, la definición de procesos críticos, equipos clínicos destinados a la atención de pacientes, sugerencias de ámbitos a supervisar y aspectos relativos a la calidad microbiológica del aire y agua.

\section{Referencias bibliográficas}

1.- Lopez-Cerero L. Papel del ambiente hospitalario y los equipamientos en la transmisión de las infecciones nosocomiales. Enferm Infecc Microbiol Clin. 2014; 32: 459-64. http://dx.doi.org/10.1016/j. eimc.2013.10.004.

2.- Wisplinghoff H, Seifert H, Wenzel R P, Edmond M B. Current trends in the epidemiology of nosocomial bloodstream infections in patients with hematological malignancies and solid neoplasms in hospitals in the United States. Clin Infect Dis 2003; 36: 1103-10. DOI: 10.1086/374339.

3.- Martino R, Ramila E, Rabella N, Muñoz J M, Peyret M, Portos J M, et al. Respiratory virus infections in adults with hematologic malignancies: a prospective study. Clin Infect Dis 2003; 36: 1-8. DOI: 10.1097/01. md.0000232560.22098.4e.

4.- Tam P Y I, Kline S, Wagner J E, Guspiel A, Streifel A, Ward G, et al. Rapidly growing mycobacteria among pediatric hematopoietic cell transplant patients traced to the hospital water supply. Pediatr Infect Dis J. 2014; 33: 1043-6. https://doi.org/10.1097/ INF.0000000000000391.

5.- Pagano L, Caira M, Candoni A, Offidani M, Fianchi L, Martino B, et al. The epidemiology of fungal infections in patients with hematologic malignancies: the SEIFEM-2004 study. Haematologica 2006; 91: 1068-75. PMID: 16885047.

6.- Morris P G, Hassan T, McNamara M, Hassan A, Wiig R, Grogan L, et al. Emergence of MRSA in positive blood cultures from patients with febrile neutropenia-a cause for concern. Support Care Cancer 2008; 16: 1085 8. DOI: 10.1007/s00520-007-0398-5.

7.- Weinstock D M,Conlon M, Iovino C, Aubrey T, Gudiol C, Riedel E, et al. Colonization, bloodstream infection, and mortality caused by vancomycin-resistant enterococcus early after allogeneic hematopoietic stem cell transplant. Biol Blood Marrow Transplant 2007; 13: 615 21. https://doi.org/10.1016/j.bbmt.2007.01.078.

8.- Cattaneo C, Quaresmini G, Casari S, Capucci M A, Micheletti M, Borlenghi E, et al. Recent changes in bacterial epidemiology and the emergence of fluoroquinoloneresistant Escherichia coli among patients with haematological malignancies: results of a prospective study on 823 patients at a single institution. J Antimicrob Chemother 2008; 61: 721-8. DOI: $10.1093 / \mathrm{jac} / \mathrm{dkm} 514$.

9.- Oliveira A L, de Souza M, Carvalho-Dias V M, Ruiz M A, Silla L, Tanaka P Y, et al. Epidemiology of bacteremia and factors associated with multi-drug-resistant gramnegative bacteremia in hematopoietic stem cell transplant recipients. Bone Marrow Transplant 2007; 39: 775-81. DOI: 10.1038/ sj.bmt.1705677.

10.- Spanik S, Krupova I, Trupl J, Kunova A, Novotny J, Mateicka F, et al. Bacteremia due to multiresistant gram-negative bacilli in neutropenic cancer patients: a case-controlled study. J Infect Chemother 1999; 5: 180-84. DOI: 10.1179/joc.1998.10.4.320.

11.- Chong Y, Shimoda S, Yakushiji H, Ito Y, Miyamoto T, Shimono N, et al. Fatal candidemia caused by azoleresistant Candida tropicalis in patients with hematological malignancies. J Infect Chemother. 2012; 18: 741-6. DOI: 10.1007/ s10156-012-0412-9.

12.- Sehulster L, Chinn R Y; CDC; HICPAC. Guidelines for environmental infection control in health-care facilities. Recommendations of CDC and the Healthcare Infection Control Practices Advisory Committee (HICPAC). MMWR Recomm Rep. 2003; 52(RR-10): 1-240. https://www.cdc.gov/ infectioncontrol/pdf/guidelines/environmentalguidelines.pdf.

13.- Marr K A, Patterson T, Denning D. Aspergillosis: pathogenesis, clinical manifestations, and therapy. Infect Dis Clin North Am 2002; 16: 875-94. PMID: 12512185.

14.- Santolaya M E, Álvarez A M, Becker A, Cofré J, Enríquez N, O`Ryan M, et al. Prospective, multicenter evaluation of risk factors associated with invasive bacterial infection in children with cancer, neutropenia, and fever. J Clin Oncol 2001; 19: 3415-21. DOI: 10.1200/ JCO.2001.19.14.3415.

15.- Rabagliati R, Bertín P, Cerón I, Rojas H, Domínguez I, Vera Á, et al. Epidemiología de neutropenia febril en pacientes adultos con leucemia aguda y linfoma. Estudio de cohorte en hospitales público y privado de Santiago, Chile. Rev Chilena Infectol 2014; 31: 721-8. http:// www.sochinf.cl/sitio/templates/sochinf2008/ documentos/2014/avances_1/14.pdf.

16.- Perdelli F, Cristina M L, Sartini M, Spagnolo A M, Dallera M, Ottria G, et al. Fungal contamination in hospital environments. Infect Control Hosp Epidemiol 2006; 27: 44-7. DOI: $10.1086 / 499149$.

17.- Panagopoulou P, Filioti J, Petrikkos G, Giakouppi P, Anatoliotaki M, Farmaki E, et al. Environmental surveillance of filamentous fungi in three tertiary care hospitals in Greece. J Hosp Infect 2002; 52: 185-91. PMID: 12419271.

18.- Fornel I, Sautor M, Lafon I, Sixt N, L'Ollivier C, Dalle F, et al. Airborne Aspergillus contamination during hospital construction works: efficacy of protective measures. Am J Infect Control 2010; 38: 189-94. DOI: 10.1016/j.ajic.2009.07.011.

19.- Alberti C, Bouakline A, Ribaud P, Lacroix C, Rousselot P, Leblanc T, et al; Aspergillus Study Group. Relationship between environmental fungal contamination and the incidence of 
invasive aspergillosis in haematology patients. $\mathrm{J}$ Hosp Infect. 2001; 48: 198-206. DOI: 10.1053/ jhin.2001.0998.

20.- Pini G, Faggi E, Donato R, Sacco C, Fanci R. Invasive pulmonary aspergillosis in neutropenic patients and the influence of hospital renovation. Mycoses. 2008; 51: 117-22. DOI: 10.1111/j.1439-0507.2007.01453.x.

21.- Lee L D, Berkheiser M, Jiang Y, Hackett B, Hachem R Y, Chemaly R F, et al. Risk of bioaerosol contamination with Aspergillus species before and after cleaning in rooms filtered with high-efficiency particulate air filters that house patients with hematologic malignancy. Infect Control Hosp Epidemiol 2007; 28: 1066-70. DOI: 10.1086/519866.

22.- Falvey D G, Streifel A J. Ten-year air sample analysis of Aspergillus prevalence in a university hospital. J Hosp Infect. 2007; 67: 35-41. DOI:10.1016/j.jhin.2007.06.008.

23.- Anaissie E, Pensak S R, Dignani C. The hospital water supply as a source of nosocomial infections. a plea of action. Arch. Intern Med. 2002; 162:1 483-92. PMID: 12090885.

24.- O'Neill E, Humphreys H. Surveillance of hospital water and primary prevention of nosocomial legionellosis: what is the evidence? J Hosp Infect. 2005; 59: 273-9. DOI: 10.1016/j. jhin.2004.09.031.

25.- Merlani G M, Francioli P. Established and emerging waterborne nosocomial infections. Curr Opin Infect Dis 2003; 16: 343-7. DOI: 10.1097/01.qco.0000083565.72029.6e.

26.- Phillips M S, von Reyn C F. Nosocomial infections due to nontuberculous mycobacteria. Clin Infect Dis. 2001; 33: 1363-74. DOI: $10.1086 / 323126$.

27.- Anaissie E J, Stratton S L, Dignani M C, Lee C K, Summerbell R C, Rex J H, et al. Pathogenic molds (including Aspergillus species) in hospital water distribution systems: a 3-year prospective study and clinical implications for patients with hematologic malignancies. Blood 2003; 101: 2542-6. DOI: 10.1182/ blood-2002-02-0530.

28.- Mesquita-Rocha S, Godoy Martínez P, Gonçalves S, Urrutia Milton D, Carlesse F, Seber A, et al. The water supply system as a potential source of fungal infection in paediatric haematopoietic stem cell units. BMC Infect Dis 2013, 13:289 DOI: 10.1186/1471-2334-13-289.

29.- Palmore T N, Stock F, White M, Bordner M A, Michelin A, Bennett J E, et al. A cluster of nosocomial legionnaire's disease linked to a contaminated hospital decorative water fountain. Infect Control Hosp Epidemiol 2009; 30: 764-8. DOI: 10.1086/598855

30.- Pasquarella C, Veronesi I, Castiglia P, Liguori G, Montagna M T, Napoli C, et al. Italian multicentre study on microbial environmental contamination in dental clinics: a pilot study. Sci Total Environ 2010; 408: 4045-51.
DOI: 10.1016/j.scitotenv.2010.05.010.

31.- Johnson D, Lineweaver L, Maze L M. Patient's bath basins as potential sources of infection: a multicenter sampling study. Am J Crit Care 2009; 18: 31-8. DOI:10.4037/ajcc2009968.

32.- Ciesielski C, Blaser M, Wang W. Role of stagnation and obstruction of water flow in isolation of Legionella pneumophila from hospital plumbing. Applied Environ Microbiol 1984; 48: 984-7 PMID: 6508313.

33.- Tomblyn M, Chiller T, Einsele H, Gress R, Sepkowitz K, Storek K, et al. Guidelines for preventing infectious complications among hematopoietic cell transplantation recipients: A global perspective. Biol Blood Marrow Transplant 2009; 15: 1143-238. DOI: 10.1016/j. bbmt.2009.06.019.

34.- Yokoe D, Casper C, Dubberke E, Lee G, Muñoz P, Palmore T, et al. Infection prevention and control in health-care facilities in which hematopoietic cell transplant recipients are treated. Bone Marrow Transplant 2009; 44: 495-507 DOI: 10.1038/bmt.2009.261.

35.- Dykewicz C A. Hospital infection control in hematopoietic stem cell transplant recipients. Emerg infect Dis 2001; 7: 263-7. DOI: 10.3201/ eid0702.700263.

36.- Sullivan K M, Dykewicz C A, Longworth D L, Boeckh M, Baden L R, Rubin R H, et al. Preventing opportunistic infections after hematopoietic stem cell transplantation: The Centers for Disease Control and Prevention, Infectious Diseases Society of America, and American Society for Blood and Marrow Transplantation Practice Guidelines and beyond. Hematology Am Soc Hematol Educ Program. 2001: 392-421. PMID: 11722995.

37.- Planning, Design, and Construction of Health Care Facilities, 2009. Second Edition. JCI resources. Disponible en: http://www. jointcommissioninternational.org/assets/1/14/ PDC09_Sample_Pages.pdf.

38.- Bambarén C, Alatrista S. Programa Médico Arquitectónico para el Diseño de Hospitales Seguros. 2008, $1^{\text {a }}$ ed. Lima, Perú. Disponible en http://bvs.ogdn.minsa.gob.pe/digitalizacion/pdf/ doc141/doc141-contenido.pdf.

39.- Mansour D, Simcock R, Gilbert D C. Acute oncology service: assessing the need and its implications. Clin Oncol (R Coll Radiol) 2011; 23: 168-73. PMID: 21465697.

40.- Kovacs J. 3 Must-haves in modern cancer center design. Healthcare Design Magazine May 2013. Disponible en: http://www. healthcaredesignmagazine.com/blogs/jenniferkovacs-silvis/3-must-haves-modern-cancerfacility-design.

41.- van de Glind I, de Roode S, Goossensen A. Do patients in hospitals benefit from single rooms? A literature review. Health Policy. 2007; 84: 153-61. DOI: 10.1016/j.healthpol.2007.06.002.

42.- Rowlands J, Noble S. How does the environment impact on the quality of life of advanced cancer patients? A qualitative study with implications for ward design. Palliat Med. 2008; 22: 768-7. DOI: $10.1177 / 0269216308093839$.

43.- Cepeda JA, Whitehouse T, Cooper B, Hails J, Jones K, Kwaku F, et al. Isolation of patients in single rooms or cohorts to reduce spread of MRSA in intensive-care units: prospective two-centre study. Lancet 2005; 365: 295-304. DOI: 10.1016/S0140-6736(05)17783-6.

44.- Schroer J. Designing uniquely responsive cancer care environments - Part 1: The need and the patient. Nov 2013. Disponible en: http://www.hksinc.com/insight/designinguniquely-responsive-cancer-care-environmentspart-1-the-need-and-the-patient/?lang=es.

45.- Lorenz S G, Dreher H M. Hospital room design and health outcomes of the aging adult. HERD. 2011; 4: 23-35. PMID: 21465433.

46.- Kibbler C C, Quick A, O'Neill A M. The effect of increased bed numbers on MRSA transmission in acute medical wards. J Hosp Infect 1998; 39: 213-9. PMID: 9699141.

47.- Bracco D, Dubois M J, Bouali R, Eggimann P. Single rooms may help to prevent nosocomial bloodstream infection and cross-transmission of methicillin-resistant Staphylococcus aureus in intensive care units. Intensive Care Med. 2007; 33: 836-40. DOI: 10.1007/s00134-007-0559-5.

48.- Teltsch D Y, Hanley J, Loo V, Goldberg P, Gursahaney A, Buckeridge D L. Infection acquisition following intensive care unit room privatization. Arch Intern Med. 2011; 171: 32 8. DOI: 10.1001/archinternmed.2010.46.

49.- Ruiz-Camps I, Aguado J M, Almirante B, Bouza E, Barbera C F, Len O, et al. Recommendations of the Spanish Society of Infectious Diseases and Clinical Microbiology (SEIMC) on the prevention of invasive fungal infection due to filamentous fungi. Enferm Infecc Microbiol Clin. 2010; 28: 172.e1-172. e21. DOI: 10.1016/j.eimc.2009.11.007.

50.- Protective Environment (Rooms). In: Guidelines for design and construction of health care facilities. The facilities guidelines institute 2006 The American Institute of Architects 1735 New York Avenue, NW Washington, DC. Disponible en: https://www.fgiguidelines.org/wp-content/ uploads/2016/07/2006guidelines.pdf.

51.- Manual del estándar general de acreditación para prestadores institucionales de atención cerrada. Superintendencia de Salud. Gobierno de Chile. Disponible en: http://www.supersalud. gob.cl/portal/articles-4530_Manual_AC_pdf. pdf.

52.- Zuckermann J, Moreira L B, Stoll P, Moreira L M, Kuchenbecker R S, Polanczyk C A. Compliance with a critical pathway for the management of febrile neutropenia and impact on clinical outcomes. Ann Hematol 2008; 87: 139-45.DOI: 10.1007/s00277-007-0390-7. 
53.- Perron T, Emara M, Ahmed S. Time to antibiotics and outcomes in cancer patients with febrile neutropenia BMC Health Serv Res 2014, 14: 162. DOI: 10.1186/1472-6963-14162.

54.- Falvey D G, Streifel A J. Ten-year air sample analysis of Aspergillus prevalence in a university hospital. J Hosp Infect 2007; 67: 3541. DOI: 10.1016/j.jhin.2007.06.008.

55.- Morris G, Kokki M H, Anderson K, Richardson $\mathrm{M}$ D. Sampling of Aspergillus spores in air. J Hosp Infect. 2000; 44: 81-92. DOI: 10.1016/j. jhin.2007.06.008.

56.- Raad I, Hanna H, Osting C, Hachem R, Umphery J, Tarrand J, et al. Masking of neutropenic patients on transport from hospital rooms is associated with a decrease in nosocomial aspergillosis during construction. Infect Control Hosp Epidemiol 2002; 23: 4143. DOI: $10.1086 / 501967$.

57.- Buttner M P, Stetzenbach L D. Monitoring airborne fungal spores in an experimental indoor environment to evaluate sampling methods and the effects of human activity on air sampling. Appl Environ Microbiol 1993; 59: 219-26. https://aem.asm.org/content/ aem/59/1/219.full.pdf.

58.- Hajjeh R A, Warnock D W. Counterpoint: invasive aspergillosis and the environmentrethinking our approach to prevention. Clin Infect Dis 2001; 33: 1549-52. DOI: $10.1086 / 322970$

59.- Oren I, Haddad N, Finkelstein R, Rowe J M. Invasive pulmonary aspergillosis in neutropenic patients during hospital construction: before and after chemoprophylaxis and institution of HEPA filters. Am J Hematol 2001; 66: 257-62. DOI: $10.1002 /$ ajh.1054.

60.- Nihtinen A, Anttila V J, Richardson M, Meri T, Volin L, Ruutu T. The utility of intensified environmental surveillance for pathogenic moulds in a stem cell transplantation ward during construction work to monitor the efficacy of HEPA filtration. Bone Marrow Transplant 2007; 40: 457-60. DOI: $10.1038 /$ sj.bmt. 1705749 .

61.- Chang C C, Ananda-Rajah M, Belcastro A, McMullan B, Reid A, Dempsey K, et al. Consensus guidelines for implementation of quality processes to prevent invasive fungal disease and enhanced surveillance measures during hospital building works, 2014. Intern Med J 2014; 44: 1389-97. DOI: 10.1111/ imj.12601.

62.- Normas para la prevención de infecciones asociadas a modificaciones estructurales y otras actividades que generan polvo ambiental en establecimientos hospitalarios, Departamento de Calidad y Seguridad del Paciente. Ministerio de Salud, Chile, mayo 2011. Disponible en: http://www.minsal.cl/portal/url/item/ a48536280e55e9f4e04001011e0163bc.pdf.
63.- Farrell I D, Barker J E, Miles E P, Hutchinson J C P. A field study of the survival of Legionella pneumophila in a hospital hot-water system. Epidemiol Infect 1990; 104: 381-7. https:// europepmc.org/backend/ptpmcrender.fcgi?accid $=$ PMC2271783\&blobtype $=$ pdf.

64.- Stout J E, Yu V L, Best M G. Ecology of Legionella pneumophila within water distribution systems. Appl Environ Microbiol 1985; 49: 221-8. PMCID: PMC238374.

65.- Sanden G N, Fields B S, Barbaree J M, Feeley $\mathrm{J}$ C. Viability of Legionella pneumophila in chlorine-free water at elevated temperatures. Curr Microbiol 1989; 18: 61-5. https://doi. org/10.1007/BF01568833.

66.- Ciesielski C A, Blaser M J, Wang W L. Role of stagnation and obstruction of water flow in isolation of Legionella pneumophila from hospital plumbing. Appl Environ Microbiol 1984; 48: 984-7. PMID: 6508313.

67.- Fields B S, Sanden G N, Barbaree J M, Morrill W E, Wadowsky R M, White E H, et al. Intracellular multiplication of Legionella pneumophila in amoebae isolated from hospital hot water tanks. Curr Microbiol 1989; 18: 131-7. https://doi.org/10.1016/09232508(91)90081-K.

68.- Kaufmann A F, McDade J E, Patton C M, Bennett J V, Skaliy P, Feeley J C, et al. Pontiac fever: isolation of the etiologic agent (Legionella pneumophila) and demonstration of its mode of transmission. Am J Epidemiol 1981; 114: 337-47. PMID: 7304569.

69.- Cristino S, Legnani P, Leoni E. Plan for the control of legionella infections in longterm care facilities: role of environmental monitoring. Intern J Hyg Environ Health 2012; 215: 279-85. DOI: 10.1016/j.ijheh.2011.08.007.

70.- Freije M. Formulating a risk reduction strategy for waterborne pathogens in hospital water systems. Am J Infect Control 2005; 33: S50-3. DOI: 10.1016/j.ajic.2005.04.004.

71.- Hosein I K, Hilla D W, Tanb T Y, Butchartc E G, Wilsond K, Finlaye G, et al. Point-ofcare controls for nosocomial legionellosis combined with chlorine dioxide potable water decontamination: a two-year survey at a welsh teaching hospital. J Hosp Infect 2005; 61: 1006. DOI: 10.1016/j.jhin.2005.02.008.

72.- Marchesi I, Marchegiano P, Bargellini A, Cencetti S, Frezza G, Miselli M, et al. Effectiveness of different methods to control legionella in the water supply: ten-year experience in an Italian university hospital. $\mathrm{J}$ Hosp Infect 2011; 77: 47-51. DOI: 10.1016/j. jhin.2010.09.012.

73.- Peiró Callizo E F, Sierra J D, Pombo J M, Baquedano C E, Huerta B P. Evaluation of the effectiveness of the pastormaster method for disinfection of legionella in a hospital water distribution system. J Hosp Infect 2005; 60: 150-8. DOI: 10.1016/j.jhin.2004.11.018.
74.- Trautmann M, Halder S, Hoegel J, Royer H, and Haller M. Point-of-use water filtration reduces endemic Pseudomonas aeruginosa infections on a surgical intensive care unit. Am J Infect Control 2008; 36: 421-9. DOI: 10.1016/j.ajic.2007.09.012.

75.- Tuttlebee C M, O'Donnell M J, Keaney, Russell R J, Sullivan D J, Falkiner F, et al. Effective control of dental chair unit waterline biofilm and marked reduction of bacterial contamination of output water using two peroxide-based disinfectants. J Hosp Infect 2002; 52: 192-205. PMID: 12419272.

76.- Vonberg R P, Eckmanns T, Bruderek J, Rüden $\mathrm{H}$, Gastmeier P. Use of terminal tap water filter systems for prevention of nosocomial legionellosis. J Hosp Infect 2005; 60: 159-62. DOI: 10.1016/j.jhin.2004.10.018.

77.- Vonberg R P, Rotermund-Rauchenberger D, Gastmeier P. Reusable terminal tap water filters for nosocomial legionellosis prevention. Ann Hematol 2005; 84: 403-5. DOI: 10.1007/ s00277-004-1000-6.

78.- Warris A, Onken A, Gaustad P, Janssen W, van der Lee H, Verweij P E, et al. Point-ofuse filtration method for the prevention of fungal contamination of hospital water. J Hosp Infect 2010; 76: 56-9. DOI: 10.1016/j. jhin.2010.03.014.

79.- O'Neill E, Humphreys H. Surveillance of hospital water and primary prevention of nosocomial legionellosis: what is the evidence? J Hosp Infect 2005; 59: 273-9. DOI: 10.1016/j. jhin.2004.09.031.

80.- L'eau dans les établissements de santé. Guide technique 2005. Ministère de la Santé et des Solidarités. Disponible en: https://solidaritessante.gouv.fr/IMG/pdf/guide_eau_dans_les_ es.pdf.

81.- Norma Calidad Del Agua Potable NCH 409/1. Disponible en: http://www.doh.gov.cl/APR/ documentos/Documents/Normas\%20NCh\%20 409\%20Calidad\%20y\%20Muestreo $\% 20$ del $\% 20$ Agua\%20Potable\%20EEO.pdf.

82.- Zubieta M, Vogel E, Celedón V, Ulloa M T, Díaz M, Catalán P, et al. Water quality characterization in 4 children's hospitals in Santiago, Chile. International Society Immunocompromised Hosts. Poster en XVIII Biennial Symposium Berlin, Germany 15-17 June 2014

83.- Pogorzelska-Maziarz M, Kalp EL. Infection prevention outside of the acute care setting: Results from the MegaSurvey of infection preventionists. Am J Infect Control 2017; 45: 597-602. http://dx.doi.org/10.1016/j. ajic.2017.03.020.

84.- Strausbaugh L J, Crossley K B, Nurse B A, Thrupp L D. Antimicrobial resistance in long-term-care facilities. Infect Control Hosp Epidemiol. 1996; 17: 129-40. PMID: 8835450.

85.- Goldrick B A. Infection control programs in 
long-term-care facilities: structure and process. Infect Control Hosp Epidemiol 1999; 20: 7649. DOI: $10.1086 / 501581$

86.- Smith P W, Bennett G, Bradley S, Drinka P, Lautenbach E, Marx J, et al. SHEA/APIC guideline: infection prevention and control in the long-term care facility, July 2008. Infect Control Hosp Epidemiol 2008; 29: 785-814. DOI: $10.1086 / 592416$.

87.- Jenkinson H, Wright D, Jones M, Dias E, Pronyszyn A, Hughes K, et al. Prevention and control of infection in non-acute healthcare settings. Nurs Stand. 2006; 20: 56-63.
88.- Rhinehart E. Infection control in home care. Emerg Infect Dis 2001; 7: 208-11. PMID: 11294708.

89.- Armstrong-Evans M, Litt M, McArthur M A, Willey B, Cann D, Liska S, et al. Control of transmission of vancomycin-resistant Enterococcus faecium in a long-term-care facility. Infect Control Hosp Epidemiol 1999; 20: 312-7. DOI: 10.1086/501623.

90.- Ben-David D, Masarwa S, Fallach N, Temkin E, Solter E, Carmeli Y, et al. Success of a national intervention in controlling carbapenem-resistant
Enterobacteriaceae in Israel's longterm care facilities. Clin Infect Dis 2018 Jul 9. DOI: 10.1093/cid/ciy572.

91.- JCI Accreditation Standards for Long Term Care, 1st Edition. Disponible en: https:// www.jointcommissioninternational.org/jciaccreditation-standards-for-long-term-care-1stedition/

92.- JCI Accreditation Standards for Long Term Care, 1st Edition. Disponible en: https:// www.jointcommissioninternational.org/jciaccreditation-standards-for-home-care-1stedition/ 